\title{
3D Discrete Dislocation Dynamics Applied to Interactions between Dislocation Walls and Particles
}

\author{
T. ZÁLEŽÁK ${ }^{a, b}$ AND A. DLOUHÝ ${ }^{a}$ \\ ${ }^{a}$ Institute of Physics of Materials, Academy of Sciences, Žižkova 22, 61662 Brno, Czech Republic \\ ${ }^{b}$ Department of Solid State Physics, Faculty of Sciences, Masaryk University, Kotlářská 2, 61137 Brno, Czech Republic \\ A 3D discrete dislocation dynamics model is presented that describes dislocation processes in crystals \\ subjected to mechanical loadings at high temperatures. Smooth and curved dislocations are approximated by a set \\ of short straight line segments. A Peach-Koehler force acting upon each segment involves all segment-to-segment \\ interactions and externally applied stress. The segment velocity is a product of a corresponding mobility and the \\ glide or climb component of the Peach-Koehler force. The model addresses interactions between dislocations and \\ rigid spherical precipitates. A migration of low angle tilt boundaries situated in a field of precipitates is simulated \\ as an example. The numerical implementation exploits symmetries of the model that yield an optimized and \\ highly efficient numerical code. Results provide detailed insight into how dislocation arrangements surmount \\ particle fields in $3 \mathrm{D}$ crystals.
}

PACS: 61.72.Ff, 61.72.Hh, 61.72.Lk, 62.20.Hg

\section{Introduction}

The discrete dislocation dynamics (DDD) is a powerful method for simulations of 3D dislocation processes. Many 3D DDD simulations deal with low temperature mechanisms where diffusion does not contribute to crystal plasticity $[1,2]$. On the other hand, only few attempts were made that extended 3D DDD to plastic deformation in the range of high temperatures, see e.g. [3]. We expect that a 3D DDD model that considers diffusion processes may represent a valuable tool yielding new insight into many fundamental issues related to creep in metallic materials. In the present study we formulate a model of this sort and apply it to migration of low angle tilt boundaries in a field of rigid precipitates.

\section{The model}

The model approximates a smooth dislocation curve by a continuous broken line composed of straight segments [4]. The linear theory of elasticity is employed and thus a local stress field results from a sum of fields due to all individual segments in the system and the externally applied stress. The total stress field at the centre of each segment then yields the Peach-Koehler force per unit length. A regularization is performed to avoid divergent terms [5]. The velocity of each segment is a product of corresponding mobility and the appropriate component of the Peach-Koehler force

$$
v_{\mathrm{C}}=\frac{D_{\mathrm{s}} \Omega}{b^{2} k T} \frac{\boldsymbol{F}_{\mathrm{C}}}{L}=B \frac{\boldsymbol{F}_{\mathrm{C}}}{L}, \quad v_{\mathrm{G}}=A \frac{\boldsymbol{F}_{\mathrm{G}}}{L},
$$

where $\Omega$ is an atomic volume, $b$ is the length of the Burgers vector, $k$ is the Boltzmann constant, $R$ is gas constant, $T$ is temperature, $D_{\mathrm{s}}=D_{0} \exp (-Q / R T)$ is the factor of self-diffusion and $Q$ is the activation energy of self-diffusion. We choose $A=10 B$ as a reasonable approximation assuming that dislocation glide is still considerably faster than climb even at fairly high temperatures. The secondary particles are represented by spheres impenetrable for the dislocations. If a dislocation segment arrives at a precipitate interface, its further motion is only possible by a combined glide and climb process, for details see [6]. Further details on our 3D DDD model are given elsewhere [7].

\section{3D simulations - low angle tilt boundaries}

As it is schematically shown in Fig. 1, we investigate a behaviour of low angle tilt boundaries acted upon by applied stress $\sigma_{x z}$. Initial dislocation spacings $h$ fall in a range $(12,34) \mathrm{nm}$. The particle structure is characterized by the particle diameter $d=100 \mathrm{~nm}$ and the interparticle spacing $\lambda=200 \mathrm{~nm}$. The edge dislocations composing the wall are parallel to the $Y$ axis and their Burgers vector $\boldsymbol{b}$ is oriented along the positive direction of the $X$ axis.

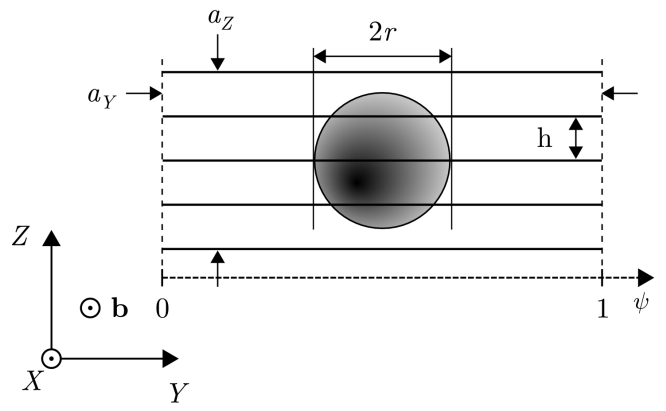

Fig. 1. A low angle tilt boundary - geometry of the simulation cell.

The system exhibits a symmetry given by two mirror planes $Y=0$ and $Z=0$. The symmetry requires that the upper- and lowermost dislocations in the computation cell (dimensions $a_{Y}$ and $a_{Z}$ ) cannot escape from their slip planes (at $Z= \pm a_{Z} / 2$ ) by climb. A particle with a diameter $d$ is placed at $[-50,0,0] \mathrm{nm}$. The periodicity 
in $Y$ and $Z$ directions is approximated by $3 \times 3$ simulation cells, which are all replicated from the central cell containing a point $[0,0,0]$. A range of applied stresses $\sigma_{x z}$ from 20 to $100 \mathrm{MPa}$ was considered in the simulations. Table shows all the relevant input parameters of the model.

Input parameters of the model.

TABLE

\begin{tabular}{c|c|c|c|c|c}
\hline \hline$\mu$ & $0.8 \times 10^{11} \mathrm{~N} \mathrm{~m}^{-2}$ & shear modulus & $N$ & 32 & \# of line segments \\
$\nu$ & 0.3 & Poisson ratio & $n$ & $\{7, \ldots, 17\}$ & $\#$ of lines in the cell \\
$D_{0}$ & $2 \times 10^{-4} \mathrm{~m}^{2} \mathrm{~s}^{-1}$ & diffusion factor & $a_{Y} ; a_{Z}$ & $200 \mathrm{~nm}$ & sim. cell dimensions \\
$Q$ & $240 \mathrm{~kJ} \mathrm{~mol}^{-1}$ & activation energy & $h$ & $a_{Z} /(n-1) \mathrm{nm}$ & initial line distance \\
$T$ & $873 \mathrm{~K}$ & temperature & $c$ & {$[-50,0,0] \mathrm{nm}$} & particle center \\
$\Omega$ & $(0.35 \mathrm{~nm})^{3}$ & atomic volume & $d$ & $100 \mathrm{~nm}$ & particle diameter \\
$\boldsymbol{b}$ & $(0.2,0,0) \mathrm{nm}$ & Burgers vector & $\lambda$ & $200 \mathrm{~nm}$ & particle distance \\
$\Delta t$ & $30 \mathrm{~ms}$ & time step & $l$ & $\langle 3,8\rangle \mathrm{nm}$ & segment length
\end{tabular}

Due to the periodic boundary conditions, local internal stresses have no effect on the initial planar form of the wall. The tilt boundary remains planar even when it moves under the action of the applied stress $\sigma_{x z}$ until first dislocation segments come into a contact with the impenetrable particles. From this moment on, the dislocation wall shape complies with the constraint imposed by particles, see Fig. 2. A further evolution can proceed in two distinct regimes, which depend on the magnitude of the applied stress $\sigma_{x z}$ and the initial spacing of dislocations in the tilt boundary $h$.

Projections into three coordinate planes in Fig. 2a characterize a shape of dislocation lines when the motion of all dislocation segments ceases. In this situation, the net driving forces acting locally on individual segments are either effectively zero (for free segments that are not in contact with particles) or are fully carried by particles (for segments interacting with particles) [6, 8]. Similar projections in Fig. 2b suggest that mutual interactions between segments are not insufficient to prevent the free dislocation lines from motion when the applied stress crosses certain threshold. The net result of the migration in this case is a system of dislocation loops stored round the particle and at least partially restored tilt dislocation boundary past the particle.

For the stable regime, Fig. 3 compares a magnitude of the Peach-Koehler forces acting on the segments in the plane $Z=a_{Z} / 2$ at the beginning of the simulation $(t=0 \mathrm{~s})$ when the external stress $\sigma_{x z}$ is applied and in the final equilibrium position of the dislocation wall $(t=105 \mathrm{~s})$. The normalized forces are plotted as a function of a parameter $\psi$ that characterizes positions of individual segments along the length of the dislocation line. It is evident that, in the logarithmic scale, forces at the equilibrium wall position are effectively zero in view of other inaccuracies associated with the numerical implementation. Results of the simulations are further summarized in Fig. 3b in a diagram that delimits three regions in a parametric plane formed by the applied
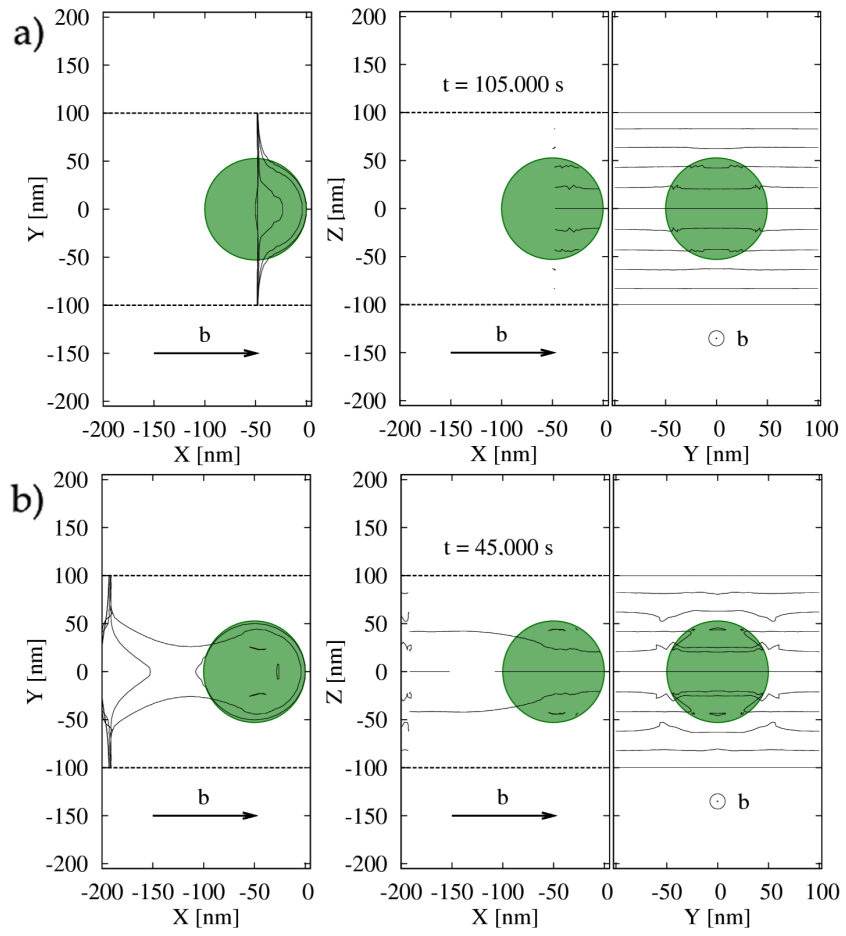

Fig. 2. An evolution of the low-angle tilt boundary with $h=20 \mathrm{~nm}$ : (a) stable configuration with $\sigma_{x z}=$ $41.2 \mathrm{MPa}, t=105 \mathrm{~s}$, (b) unstable configuration with $\sigma_{x z}=54.5 \mathrm{MPa}, t=45 \mathrm{~s}$.

stress $\sigma_{x z}$ and the dislocation spacing $h$. Combinations of applied stress and the spacing $h$ that resulted in the stable (pinned) wall configurations fall into the region PINNED. Conversely, simulations in which the original low angle tilt boundary passed by the particle field form the region PASS. Some of the particle-dislocation wall interactions belonging to the category PASS resulted in a wall disintegration (region PASS \& SPLIT). In these 

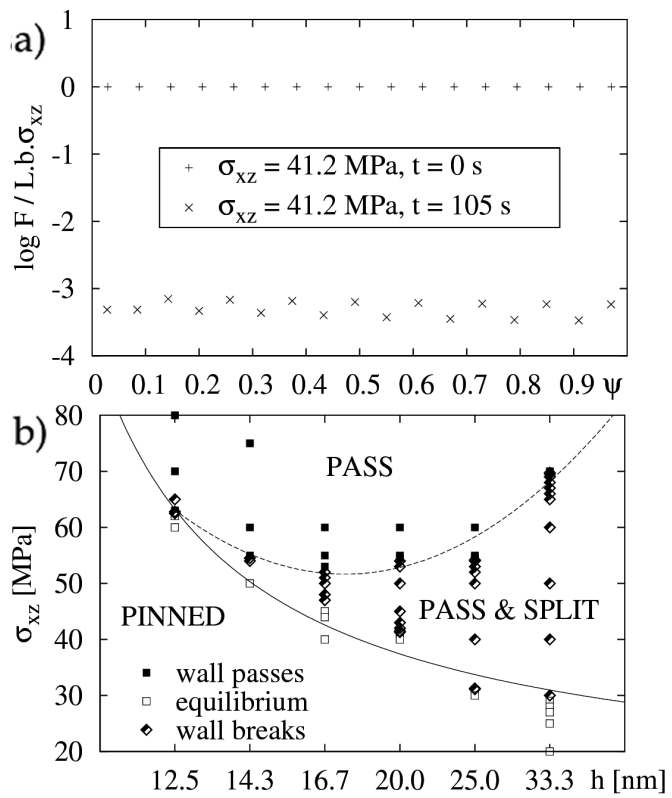

Fig. 3. (a) Force along the dislocation line in the plane $Z=a_{Z} / 2$, (b) dislocation wall regimes for varying $\sigma_{x z}$ and $h$.

cases, the tilt boundary splits during the passing process into two or more distinct boundaries with dislocation spacings $h$ larger than the original spacing $h$. Most importantly, the critical stresses demarking the stable and unstable regimes (PINNED and PASS) are approximately inversely proportional to the corresponding line spacings $h$.

\section{Discussion}

There is an ample experimental evidence showing that formation and migration of low angle dislocation boundaries is a generic process observed in many materials systems subjected to the high temperature loading, including precipitation hardened alloys [9, 10]. High sensitivity of the strain rate to the applied stress in these alloys is still not fully understood and is a subject of ongoing discussion in the scientific literature [11]. In the present study, we show that even fully flexible tilt boundaries can be pinned by the particle structure provided the combination of applied stress $\sigma_{x z}$ and the density of boundary dislocations (dislocation spacing $h<h_{\text {crit }}$ ) is within the regime PINNED shown in Fig. 3b. Consequently, the deformation process would gradually cease as the mobile dislocations stop and knit into the pinned dislocation walls.

The interactions between particles and low angle dislocation boundaries thus may give rise to a non-negligible threshold stresses even at high temperatures where dislocation climb is important. The threshold stresses of that sort measured in creep experiments have often been reported, see e.g. [9]. We note that the range of $h_{\text {crit }}$ in Fig. 3b, which corresponds to applied stresses between
20 and $60 \mathrm{MPa}$, yields tilt boundary misorientation angles from $0.3^{\circ}$ to $0.9^{\circ}$. This is in a very good agreement with the typical misorientation angles observed by TEM after high temperature deformation. The numerical implementation of our 3D DDD model thus seems to yield relevant information on the behaviour of low angle dislocation boundaries constrained by secondary particles. Further parametric studies are needed to address a range of particle sizes and interparticle spacings.

\section{Summary and conclusion}

A 3D DDD model has been numerically implemented. Interactions between dislocation tilt boundaries and impenetrable spherical particles were investigated under conditions of high temperature creep. One specific particle distribution has been considered with the particle size $d=100 \mathrm{~nm}$ and the interparticle spacing $\lambda=200 \mathrm{~nm}$. Results of the numerical simulations suggest that the migration of the dislocation tilt boundaries can be considerably restricted due to particle-boundary interactions. For a given level of externally applied stress, tilt boundaries with dislocation spacing smaller than a critical value $h_{\text {crit }}$ are fully immobilized. The values of $h_{\text {crit }}$ are inversely proportional to the applied stress.

\section{Acknowledgments}

The authors acknowledge the financial support from the Czech Science Foundation under contracts No. 202/ 09/2073 and 106/09/H035. Additional support has been received from the Ministry of Education, Youth and Sports within the project COST P19-OC 162.

\section{References}

[1] B. Devincre, L.P. Kubin, Mater. Sci. Eng. A 234-236, 8 (1997).

[2] D. Weygand, L.H. Friedman, E. van der Giessen, A. Needleman, Mater. Sci. Eng. A 309-310, 420 (2001).

[3] D. Mordehai, E. Clouet, M. Fivel, M. Verdier, Philos. Mag. 88, 899 (2008).

[4] P. Hirth, J. Lothe, Theory of Dislocations, 2nd ed., Krieger Publ. Co., Malabar 1992.

[5] W. Cai, A. Arsenlis, Ch.R. Weinberger, V.V. Bulatov, J. Mech. Phys. Sol. 54, 561 (2006).

[6] D. Holec, A. Dlouhý, Z. Metallkde 96, 558 (2005).

[7] T. Záležak, A. Dlouhý, Key Eng. Mater. 465, 115 (2011).

[8] D. Holec, B.Sc. Thesis, Masaryk University, Brno 2003.

[9] J.H. Hausselt, W.D. Nix, Acta Metall. 25, 595 (1977).

[10] W. Blum, in: Materials Science and Technology, Vol. 6, Ed. H. Mughrabi, VCH Verlagsgesellschaft, Weinheim 1993.

[11] J. Čadek, V. Šustek, M. Pahutová, Mater. Sci. Eng. A 225, 22 (1997). 